\title{
The relationship between intensity and stochastic matrices for continuous-time discrete value stochastic non homogeneous processes with Markov property
}

\author{
Mi los lawa Sokol
}

Faculty of Biology, Biological and Chemical Research Centre, University of Warsaw, Zwirki i Wigury 101,

\section{Abstract}

02-089 Warsaw, Poland

The matrices of non-homogeneous Markov processes consist of time-dependent functions whose values at time form typical intensity matrices. For solving some problems they must be changed into stochastic matrices. A stochastic matrix for non-homogeneous Markov process consists of time-dependent functions, whose values are probabilities and it depend on assumed time period. In this paper formulas for these functions are derived. Although the formula is not simple, it allows proving some theorems for Markov stochastic processes, well known for homogeneous processes, but for non-homogeneous ones the proofs of them turned out shorter.

Keywords: Markov process Non-homogeneous process Intensity matrix Stochastic matrix

\section{Introduction}

Non-homogeneous (sometimes called inhomogeneous) Markov stochastic processes often appear in scientific literature as solutions of many real problems due to twenty-four hour or seasonal fluctuations of probability of many real events [2], [4], [5], [6], [10], [11], [12], [14], [17]. But the general theory of these processes very seldom appears in textbooks of stochastic processes. The theorems in this paper apply to all continuous-time, discrete value Markov stochastic processes.

All non-homogeneous continuous-time discrete value stochastic processes $\left(X_{t}\right)_{t \in[0, T)}$ with Markov property can be defined by $\left[f_{k, n}(t)\right]_{k, n \in \mathrm{N}}$ - a matrix of time-dependent, integrable and limited on limited intervals functions $f_{k, n}:[0, T) \rightarrow \mathrm{R}$ where:

$$
\begin{gathered}
f_{k, n}(t) \geq 0 \text { forallkandnifn } \neq k, \\
f_{k, k}(t) \leq 0 \text { forall }, \\
\sum_{n \in \mathrm{N}} f_{k, n}(t)=0 \text { forall } .
\end{gathered}
$$

They can be defined by a probability space $(\Omega, \sigma(\Omega), P)$ for a Markov stochastic process $\left(X_{t}\right)_{t \in[0, T)}$ by the formulas:

$$
f_{k, n}(t)=\lim _{\tau \rightarrow 0^{+}} \frac{P\left\{X_{t+\tau}=E_{n} \mid X_{t}=E_{k}\right\}}{\tau}
$$

or

$$
f_{k, k}(t)=\lim _{\tau \rightarrow 0^{+}} \frac{P\left\{X_{t+\tau}=E_{k} \mid X_{t}=E_{k}\right\}-1}{\tau},
$$

or they can be defined by formulas without described a stochastic process, althought such process always exists ([13]).

The matrix of functions $\left[f_{k, n}(t)\right]$ is named an intensity matrix [15], [1], or state-transition matrix [8], [16] for the 
stochastic process $\left(X_{t}\right)_{t \in[0, T)}$. The intensity matrix specifies all features of continuous-time discrete value stochastic processes with Markov property. In particular it should clearly define a stochastic matrix of this process for any fixed time-period.

Idea of time-depended stochastic and intensity matrices are not new [3], [9]. The equations that I am derived in my article, can complement the theory shown in these papers. Moreover the theory of non-homogeneous stochastic processes with Markov property turned out to be easer than theory of their special case: homogeneous processes.

The first aim of this study is to construct a stochastic matrix for a continuous-time discrete value stochastic process with Markov property, whose intensity matrix (e.g. matrix of the function $f_{k, n}(t)$ wchich satisfy the properties 1,2

and 3) and time period $\Delta$ is known. The formulas of probability are the same than in [9] but written in different way. This simplifies and shortens the proofs of some theorems about Markov processes and it is the second aim. The third aim is showing that for homogeneous processes similar formulas are too complicated for applications. It shows that Markov stochastic processes theory should be taught starting with non-homogeneous processes.

\section{An intensity matrix for the stochastic matrices}

For non-homogeneous stochastic processes, stochastic probability matrices $\left[F_{k, n}\left(t_{1}, t_{2}\right)\right]_{k, n \in \mathrm{N}}$ can be different for different $t_{1}$ and $t_{2}$ such that $t_{1}<t_{2}$ are initial and final times of one or multiple transitions from state $E_{k}$ to state $E_{n}$. For these matrices the following features have been assumed:

$$
\begin{gathered}
0 \leq F_{k, n}\left(t_{1}, t_{2}\right) \leq 1 \text { forallkandn, } \\
\sum_{n \in \mathrm{N}} F_{k, n}\left(t_{1}, t_{2}\right)=1 \text { forallk, } \\
\lim _{\tau \rightarrow 0} F_{k, n}(t, t+\tau)=0 \text { forall } \neq k
\end{gathered}
$$

and

$$
\begin{gathered}
\lim _{\tau \rightarrow 0} F_{k, k}(t, t+\tau)=1 \text { forall }, \\
\lim _{\tau \rightarrow 0} \frac{F_{k, n}(t, t+\tau)-F_{k, n}(t, t)}{\tau}=f_{k, n}(t) \text { forallnand } k
\end{gathered}
$$

where $f_{k, n}(t)$ are integrable functions. $F_{k, n}(t, t)=0$ for $k \neq n$ and $F_{k, k}(t, t)=1$ for all $k$.

For a Markov stochastic process the following formula is true:

$$
F_{k, n}\left(t_{1}, t_{3}\right)=\sum_{m \in \mathbb{N}} F_{k, m}\left(t_{1}, t_{2}\right) F_{m, n}\left(t_{2}, t_{3}\right)
$$

for all $k$ and $n$, and $t_{1}<t_{2}<t_{3}$. In the proof of this equation the property (7) is used. But there isn't a proof that the property $(7)$ is nessesary.

After the division of the interval $\left[t_{1}, t_{2}\right)$ into $L+1$ equal segments $\left[t_{1}+i \delta, t_{1}+(i+1) \delta\right.$ ) where $\delta=\frac{t_{2}-t_{1}}{L+1}$, we receive the formula: 


$$
\begin{gathered}
F_{k, n}\left(t_{1}, t_{2}\right)= \\
\sum_{m_{1} \in N} \cdots \sum_{m_{L} \in \mathrm{N}} F_{k, m}\left(t_{1}, t_{1}+\delta\right) F_{m_{1}, m_{2}}\left(t_{1}+\delta, t_{1}+2 \delta\right) \cdots F_{m_{L}, n}\left(t_{1}+L \delta, t_{2}\right) .
\end{gathered}
$$

In many sequences $\left(k, m_{1}, \ldots, m_{L}, n\right)$ the equalities: $k=m_{1}$ or $m_{i}=m_{i}+1$, or $m_{L}=n$ can be observed. Each such a long sequence can be ordered by number of state changes, noted as $r$. Sometimes it will be note $k=m_{0}$, $t_{1}=x_{0}$ and $n=m_{r}, t_{2}=x_{r+1}$ for reduction of the notation of the equations.

Let $\left(x_{1}, x_{2}, \ldots x_{r}\right)$ be beginnigs of intervals $[i \delta,(i+1) \delta)$ of changing states $E_{k}=E_{m_{0}}$ to $E_{m_{1}}, E_{m_{1}}$ to $E_{m_{2}} \cdots$, $E_{m_{r-1}}$ to $E_{m_{r}}=E_{n}$. The interval $\left[x_{0}, x_{1}\right)$ is a time of no changing state $E_{m_{0}}=E_{k}$.

Equation (11) can be noted as:

$$
\begin{gathered}
F_{k, n}\left(t_{1}, t_{2}\right)= \\
\sum_{r=0}^{L} \sum_{m_{1} \neq m_{2} \neq \cdots \neq m_{r-1} x_{1}<x_{2}<\cdots<x_{r}} G_{k, k}\left(t_{1}, x_{1}\right) G_{k, m_{1}}\left(x_{1}, x_{2}\right) \cdots G_{m_{r-1}, n}\left(x_{r}, t_{2}\right)
\end{gathered}
$$

where:

$$
\begin{gathered}
G_{k, k}\left(t_{1}, x_{1}\right)=\prod_{i=0}^{\left(x_{1}-t_{1}\right) / \delta-1} F_{k, k}\left(x_{0}+i \delta, x_{0}+(i+1) \delta\right), \\
G_{m_{j-1}, m_{j}}\left(x_{j}, x_{j+1}\right)= \\
F_{m_{j-1}, m_{j}}\left(x_{j}, x_{j}+\delta\right) \prod_{i=1}^{\left(x_{j+1}-x_{j}\right) / \delta-1} F_{m_{j}, m_{j}}\left(x_{j}+i \delta, x_{j}+(i+1) \delta\right)
\end{gathered}
$$

for $j=1,2, \ldots, r$.

For $r=0$ and $k=n$ this equation is reduced to $G_{k, k}\left(t_{1}, t_{2}\right)$. For $r=0$ and $k \neq n$ it is equal 0 , as probability of impossible event. The event $r=1$ and $k=n$ is also impossible, so it's probability is equal 0 .

Theorem For $r=0$ : 


$$
\lim _{\delta \rightarrow 0} G_{k, k}\left(t_{1}, t_{2}\right)=\exp \left(\int_{t_{1}}^{t_{2}} f_{k, k}(x) d x\right)
$$

For $\mathrm{r}=1$ and $n \neq k$ :

$$
\lim _{\delta \rightarrow 0} \sum_{x} G_{k, k}\left(t_{1}, x\right) G_{k, n}\left(x, t_{2}\right)=\int_{t_{1}}^{t_{2}} f_{k, n}(x) \exp \left(\int_{t_{1}}^{x} f_{k, k}(s) d s+\int_{x}^{t_{2}} f_{n, n}(s) d s\right) d x .
$$

For $r>1$ transitions and $k \neq m_{1} \neq \cdots \neq m_{r-1} \neq n$ :

$$
\begin{gathered}
\lim _{\delta \rightarrow 0} \sum_{x} G_{k, k}\left(t_{1}, x_{1}\right) G_{k, m_{1}}\left(x_{1}, x_{2}\right) \cdots G_{m_{r-1}, n}\left(x_{r}, t_{2}\right)= \\
\int_{t_{1}}^{t_{2} \int_{1} t_{2}} \ldots \int_{x_{r-1}}^{t_{2}} f_{k, m_{1}}\left(x_{1}\right) f_{m_{1}, m_{2}}\left(x_{2}\right) \ldots f_{m_{r-1}, n}\left(x_{r}\right) . \\
\exp \left(\int_{t_{1}}^{x_{1}} f_{k, k}(s) d s+\int_{x_{1}}^{x_{2}} f_{m_{1}, m_{1}}(s) d s+\ldots+\int_{x_{r}}^{t_{2}} f_{n, n}(s) d s\right) d x_{r} \ldots d x_{2} d x_{1} .
\end{gathered}
$$

Proof Using $s_{i}=t_{1}+i \delta$ the following conversions can be done:

$$
\begin{gathered}
\lim _{\delta \rightarrow 0} G_{k, k}\left(t_{1}, x_{1}\right)=\lim _{\delta \rightarrow 0} \prod_{i=0}^{\left(x_{1}-t_{1}\right) / \delta-1} F_{k, k}\left(s_{i}, s_{i}+\delta\right)= \\
\lim _{\delta \rightarrow 0} \exp \left(\sum_{i=0}^{\left(x_{1}-t_{1}\right) / \delta-1} \ln \left(F_{k, k}\left(s_{i}, s_{i}+\delta\right)\right)\right)= \\
\exp \lim _{\delta \rightarrow 0}\left(\delta \sum_{i=0}^{\left(x_{1}-t_{1}\right) / \delta-1} \frac{\ln \left(F_{k, k}\left(s_{i}, s_{i}+\delta\right)\right)}{F_{k, k}\left(s_{i}, s_{i}+\delta\right)-1} \frac{F_{k, k}\left(s_{i}, s_{i}+\delta\right)-1}{\delta}\right)= \\
\exp \left(\int_{t_{1}}^{x_{1}} f_{k, k}(x) d x\right)
\end{gathered}
$$

because of $\lim _{x \rightarrow 1} \ln (x) /(x-1)=1$ and $\lim _{\delta \rightarrow 0}\left(F_{k, k}(t, t+\delta)-1\right) / \delta=f_{k, k}(t)$ due to assumption (6).

After substituting $x_{1}=t_{2}$ equation (15) is received. Using $s_{i}=x+i \delta$ the following conversions can be done:

$$
\lim _{\delta \rightarrow 0} \sum_{x=t_{1}}^{t_{2}} G_{k, k}\left(t_{1}, x\right) G_{k, n}\left(x, t_{2}\right)=
$$




$$
\begin{gathered}
\lim _{\delta \rightarrow 0} \sum_{x=t_{1}}^{t_{2}} G_{k, k}\left(t_{1}, x\right) F_{k, n}(x, x+\delta) \prod_{i=0}^{\left(t_{2}-x\right) / \delta-1} F_{k, k}\left(s_{i}, s_{i}+\delta\right)= \\
\lim _{\delta \rightarrow 0} \sum_{x=t_{1}}^{t_{2}} G_{k, k}\left(t_{1}, x\right) F_{k, n}(x, x+\delta) \exp \left(\sum_{i=0}^{\left(t_{2}-x\right) / \delta-1} \ln \left(F_{k, k}\left(s_{i}, s_{i}+\delta\right)\right)\right)= \\
\lim _{\delta \rightarrow 0} \delta \sum_{x=t_{1}}^{t_{2}} G_{k, k}\left(t_{1}, x\right) \frac{F_{k, n}(x, x+\delta)}{\delta} \exp \left(\delta \sum_{i=0}^{\left(t_{2}-x\right) / \delta-1} \frac{\ln \left(F_{k, k}\left(s_{i}, s_{i}+\delta\right)\right)}{\delta}\right)= \\
\lim _{\delta \rightarrow 0} \int_{t_{1}}^{t_{2}} \exp \left(\int_{t_{1}}^{x} f_{k, k}(s) d s\right) f_{k, n}(x) \exp \left(\int_{x}^{t_{2}} f_{k, k}(s) d s\right) .
\end{gathered}
$$

The last equation can be transformed into (16). The general equation for $r$ transitions can be proved by mathematical inductions using the transformations shown above.

According to theorem 1 the general relationship between an intensity matrix and a stochastic matrix is shown by the formula:

$$
F_{k, n}\left(t_{1}, t_{2}\right)=\sum_{r=0}^{\infty} \sum_{m_{1} \neq k m_{2} \neq m_{1}} \ldots \sum_{m_{r-1} \neq m_{r-2}} H_{m_{r-1} \neq n}^{t_{1}, r}\left(k, m_{1}, m_{2}, \ldots, m_{r-1}, n\right)
$$

where:

$$
\begin{aligned}
& H_{t_{1}, 0}^{t_{2}}(k)=\exp \left(\int_{t_{1}}^{t_{2}} f_{k, k}(x) d x\right) \\
& H_{t_{1}, 1}^{t_{2}}(k, n)=\int_{t_{1}}^{t_{2}} f_{k, n}(x) \exp \left(\int_{t_{1}}^{x} f_{k, k}(s) d s+\int_{x}^{t_{2}} f_{n, n}(s) d s\right) d x, \\
& H_{x_{0}, r}^{x_{r+1}}\left(m_{0}, m_{1}, \ldots, m_{r}\right)= \\
& \int_{x_{0}}^{x_{r+1}} \int_{x_{1}}^{x_{r+1}} \ldots \int_{x_{r-1}}^{x_{r+1}} \prod_{i=1}^{r} f_{m_{i-1}, m_{i}}\left(x_{i}\right) \exp \left(\sum_{i=0}^{r} \int_{x_{i}}^{x_{i+1}} f_{m_{i}, m_{i}}(s) d s\right) d x_{r} \ldots d x_{2} d x_{1}
\end{aligned}
$$

where $x_{0}=t_{1}, x_{r+1}=t_{2}, m_{0}=k$ and $m_{r}=n$. They are integrals of the functions which are known in statistics as incomplete integrals. They are not new concepts in mathematics, but rarely used. 


\section{Stochastic matrices for the intensity matrix}

In this section the matrix $\left[f_{k, n}(t)\right]_{k, n \in \mathrm{N}}$ of integrable functions with properties (1), (2) and (3) given in introduction. Matrix $\left[F_{k, n}\left(t_{1}, t_{2}\right)\right]$ is defined by formulas (20)-(23). The properties of this matrix are analysed.

Property (1) is obvious. Properties (3) and (6) are true if each $F_{k, n}\left(t_{1}, t_{2}\right)$ is finite. Each $F_{k, n}\left(t_{1}, t_{2}\right)$ is finite if the sum $\sum_{n=1}^{\infty} F_{k, n}\left(t_{1}, t_{2}\right)$ is finite. So it is enough to analyse property (2).

Lemma For any $x_{1}$ and $x_{2}\left(x_{1}<x_{2}\right)$ and time-dependent, integrable function $f$

$$
\int_{y_{1}}^{y_{2}} f(x) \exp \left(\int_{y_{1}}^{x} f(s) d s\right) d x=\exp \left(\int_{y_{1}}^{y_{2}} f(x) d x\right)-1
$$

Proof Let $h(x)=\exp \int_{y_{1}}^{x} f(s) d s$. Then: $h^{\prime}(x)=f(x) \exp \left(\int_{y_{1}}^{x} f(s) d s\right)$. So:

$$
\int_{y_{1}}^{y_{2}} f(x) \exp \left(\int_{y_{1}}^{x} f(s) d s\right) d x=h\left(y_{2}\right)-h\left(y_{1}\right)=\exp \left(\int_{y_{1}}^{y_{2}} f(x) d x\right)-1 \text {. }
$$

Theorem Let $\left[f_{k, n}(t)\right]_{k, n \in \mathrm{N}}$ be any finite or infinite matrix of integrable functions, finite on finite intervals of time, which has properties (7), (8) and (9). Then the matrix of the functions given by formula (20)-(23) satisfy the property:

$$
\sum_{n \in \mathbb{N}} F_{k, n}\left(t_{1}, t_{2}\right) \leq 1
$$

for any time period $\left(t_{1}, t_{2}\right)$.

Proof It will be proved that for any $q>1$ :

$$
\sum_{n} \sum_{r=0}^{q-1} \sum_{m_{1} \neq k} \sum_{m_{2} \neq m_{1}} \ldots \sum_{m_{r-1} \neq m_{r-2}} H_{m_{r-1} \neq n}^{t_{2}, r}\left(k, m_{1}, m_{2}, \ldots, m_{r-1}, n\right) \leq 1
$$

This inequolity is the same, as:

$$
\sum_{r=0}^{q-1} \sum_{m_{1} \neq k} \sum_{m_{2} \neq m_{1}} \ldots \sum_{m_{r-1} \neq m_{r-2}} \sum_{m_{r} \neq m_{r-1}} H_{t_{1}, r}^{t_{2}}\left(k, m_{1}, m_{2}, \ldots, m_{r-1}, m_{r}\right) \leq 1
$$

Let $q>1$. Let for $x_{0}=t_{1}$ and $x_{q+1}=t_{2}$, and $m_{0}=k$.

$$
K_{x_{0}, q}^{x_{q+1}}\left(m_{0}, m_{1}, \ldots, m_{q}\right)=
$$




$$
\int_{x_{0}}^{x_{q+1}} \int_{x_{1}}^{x_{q+1}} \ldots \int_{x_{q-1}}^{x_{q+1}} \prod_{i=1}^{q} f_{m_{i-1}, m_{i}}\left(x_{i}\right) \exp \left(\sum_{i=0}^{q-1} \int_{x_{i}}^{x_{i+1}} f_{m_{i}, m_{i}}(s) d s\right) d x_{q} \ldots d x_{2} d x_{1}
$$

There is a difference between $K_{x_{0}, q}^{x_{q+1}}\left(m_{0}, m_{1}, \ldots, m_{q}\right)$ and $H_{x_{0}, q}^{x_{q+1}}\left(m_{0}, m_{1}, \ldots, m_{q}\right)$ because the sum of components of the function $\exp ()$ in $K_{x_{0}, q}^{x_{q+1}}\left(m_{0}, m_{1}, \ldots, m_{q}\right)$ is shorter than in $H_{x_{0}, q}^{x_{q+1}}\left(m_{0}, m_{1}, \ldots, m_{q}\right)$.

According to assumption (9):

$$
\begin{gathered}
\sum_{m_{q} \neq m_{q-1}} K_{x_{0}, q}^{x_{q+1}}\left(m_{0}, m_{1}, \ldots, m_{q}\right)= \\
-\int_{x_{0}}^{x_{q+1}} \int_{x_{1}}^{x_{q+1}} \ldots \int_{x_{q-2}}^{x_{q+1}} \prod_{i=1}^{q-1} f_{m_{i-1}, m_{i}}\left(x_{i}\right) \exp \left(\sum_{i=0}^{q-2} \int_{x_{i}}^{x_{i+1}} f_{m_{i}, m_{i}}(s) d s\right) W\left(x_{q-1}\right) d x_{q-1} \ldots d x_{2} d x_{1}
\end{gathered}
$$

where:

$$
\begin{gathered}
W\left(x_{q-1}\right)=\int_{x_{q-1}}^{x_{q+1}} f_{m_{q-1}, m_{q-1}}\left(x_{q}\right) \exp \left(\int_{x_{q-1}}^{x_{q}} f_{m_{q-1}, m_{q-1}}(s) d s\right) d\left(x_{q}\right)= \\
\exp \left(\int_{x_{q-1}}^{x_{q+1}} f_{m_{q-1}, m_{q-1}}(s) d s\right)-1
\end{gathered}
$$

according to lemma 1. After substituting this formula into equation (29) we receive:

$$
\begin{gathered}
\sum_{m_{q} \neq m_{q-1}} K_{t_{1}, q}^{t_{2}}\left(m_{0}, m_{1}, \ldots, m_{q}\right)= \\
-H_{t_{1}, q-1}^{t_{2}}\left(m_{0}, m_{1}, \ldots, m_{q-1}\right)+K_{t_{1}, q-1}^{t_{2}}\left(m_{0}, m_{1}, \ldots, m_{q-1}\right) .
\end{gathered}
$$

It can be proved that:

$$
\begin{gathered}
\sum_{m_{1} \neq m_{0}} \sum_{m_{2} \neq m_{1}} \ldots \sum_{m_{q} \neq m_{q-1}} K_{t_{1}, q}^{t_{2}}\left(m_{0}, m_{1}, \ldots, m_{q}\right)= \\
=-\sum_{r=1}^{q-1} \sum_{m_{1} \neq m_{0}} \sum_{m_{2} \neq m_{1}} \ldots \sum_{m_{r-1} \neq m_{r-2}} H_{t_{1}, r}^{t_{2}}\left(m_{0}, m_{1}, \ldots, m_{r}\right)-
\end{gathered}
$$




$$
\begin{gathered}
\exp \left(\int_{t_{1}}^{t_{2}} f_{m_{0}, m_{0}}(s) d s\right)+1 \\
=-\sum_{r=0}^{q-1} \sum_{m_{1} \neq m_{0}} \sum_{m_{2} \neq m_{1}} \ldots \sum_{m_{r} \neq m_{r-1}} H_{t_{1}, r}^{t_{2}}\left(m_{0}, m_{1}, \ldots, m_{r}\right)+1
\end{gathered}
$$

So:

$$
\begin{gathered}
\sum_{r=0}^{q-1} \sum_{m_{1} \neq m_{0}} \sum_{m_{2} \neq m_{1}} \ldots \sum_{m_{r} \neq m_{r-1}} H_{t_{1}, r}^{t_{2}}\left(m_{0}, m_{1}, \ldots, m_{r}\right)= \\
1-\sum_{m_{1} \neq m_{0}} \sum_{m_{2} \neq m_{1}} \ldots \sum_{m_{q} \neq m_{q-1}} K_{t_{1}, q}^{t_{2}}\left(m_{0}, m_{1}, \ldots, m_{q}\right) \leq 1
\end{gathered}
$$

The $q$ can be replaced by $\infty$. It means that $\sum_{n} F_{k, n}\left(t_{1}, t_{2}\right) \leq 1$.

Theorem Let $\left[f_{k, n}(t)\right]_{k, n \in \mathrm{N}}$ be any matrix of integrable functions, which has properties (7), (8) and (9), and there exists such a real number $M$, that $f_{k, k}(t)>-M$ for each $k$ and time $t$. Then:

$$
\sum_{n} F_{k, n}\left(t_{1}, t_{2}\right)=1 \text { forallkandtimeperiod }\left[t_{1}, t_{2}\right) .
$$

Proof Due to equation (33) it is sufficient to prove that:

$$
\lim _{q \rightarrow \infty} \sum_{m_{1} \neq m_{0}} \ldots \sum_{m_{q-1} \neq m} \sum_{q-2} K_{q} \neq m_{q-1}^{t_{2}}\left(m_{1}, q, m_{1}, \ldots, m_{q}\right)=0 .
$$

For any q:

$$
\begin{gathered}
\sum_{m_{1} \neq k} \ldots \sum_{m_{q-1} \neq m_{q-2}} \sum_{m^{\neq m}} K_{q-1}^{t_{2}}\left(m_{1}, m_{1}, \ldots, m_{q}\right)= \\
\sum_{m_{1} \neq k} \ldots \sum_{m_{q-1} \neq m_{q-2} m_{q} \neq m_{q-1} t_{1} x_{1}} \int_{x_{q-1}}^{t_{2} t_{2}} \ldots \int_{m_{0}, m_{1}}^{t_{2}}\left(x_{1}\right) \ldots f_{m_{q-1}, m_{q}}\left(x_{q}\right) \\
\exp \left(\int_{t_{1}}^{x_{1}} f_{m_{0}, m_{0}}(s) d s+\ldots+\int_{x_{q}-1}^{x_{q}} f_{m_{q-1}, m_{q-1}}(s) d s\right) d x_{q} \ldots d x_{1}=
\end{gathered}
$$




$$
\begin{aligned}
& \int_{t_{1}}^{t_{2}} \int_{x_{1}}^{t_{2}} \ldots \int_{x_{q-1}}^{t_{2}}(-1) f_{m_{0}, m_{0}}\left(x_{1}\right) \ldots(-1) f_{m_{q-1}, m_{q-1}}\left(x_{q}\right) \\
& \exp \left(\int_{t_{1}}^{x_{1}} f_{m_{0}, m_{0}}(s) d s+\ldots+\int_{x_{q}-1}^{x_{q}} f_{m_{q-1}, m_{q-1}}(s) d s\right) d x_{q} \ldots d x_{1} \leq \\
& M^{q} \int_{t_{1}}^{t_{2} x_{1}} \int_{x_{q-1}}^{t_{2}} \ldots \int_{x_{2}}^{t_{2}} \exp \left(-M\left(x_{1}-t_{1}\right)-\ldots-M\left(x_{q}-x_{q-1}\right)\right) d x_{q} \ldots d x_{1}= \\
& M^{q} \int_{t_{1}}^{t_{2} \int_{1} \int_{2}} \ldots \int_{x_{q-1}}^{t_{2}} \exp \left(-M\left(x_{q}-t_{1}\right)\right) d x_{q} \ldots d x_{1}= \\
& M^{q-1} \int_{t_{1}}^{t_{2} \int_{1}} \int_{x_{1}} \ldots \int_{x_{q-2}}^{t_{2}} \exp \left(-M\left(x_{q-1}-t_{1}\right)\right) d x_{q-1} \ldots d x_{1}- \\
& M^{q-1} \exp \left(-M\left(t_{2}-t_{1}\right)\right) \int_{t_{1}}^{t_{2} x_{1}} \int_{x_{q-2}}^{t_{2}} \ldots \int_{x^{2}} d x_{q-1} \ldots d x_{1}= \\
& M^{q-1} \int_{t_{1}}^{t_{2} \int_{1}} \int_{x_{1}} \ldots \int_{x_{q-2}}^{t_{2}} \exp \left(-M\left(x_{q-1}-t_{1}\right)\right) d x_{q-1} \ldots d x_{1}- \\
& M^{q-1} \exp \left(-M\left(t_{2}-t_{1}\right)\right) \frac{\left(t_{2}-t_{1}\right)^{q-1}}{(q-1) !}=\ldots= \\
& 1-\sum_{i=0}^{q-1} \exp \left(-M\left(t_{2}-t_{1}\right)\right) \frac{\left(M\left(t_{2}-t_{1}\right)\right)^{q-1-i}}{(q-1-i) !}
\end{aligned}
$$

But $\sum_{r=0}^{\infty} \frac{\lambda^{j}}{j !} \exp (-\lambda)=1$ for any $\lambda$, so the equation 35 is true.

If the matrix $\left[f_{k, n}(t)\right]_{k, n \in I}$ is finite (e.g. the set of indexes $I$ is finite) and all functions $f_{k, n}$ are limited, then there 
exists a real number $M$, such that $f_{n, n}(t)>-M$ for all $\mathrm{n}$. For each finite intensity matrix $\left[f_{k, n}(t)\right]_{k, n \in I}$ the corresponding stochastic matrix $\left[F_{k, n}\left(t_{1}, t_{2}\right)\right]_{k, n \in I}$ has the property $\sum_{n} F_{k, n}\left(t_{1}, t_{2}\right)=1$ for all $k$ and time period $\left[t_{1}, t_{2}\right)$.

Property $\sum_{n} F_{k, n}\left(t_{1}, t_{2}\right)=1$ isn't true for some infinite matrices $\left[f_{k, n}(t)\right]_{k, n \in \mathrm{N}}$. It has long been known that there exist stochastic processes which stochastic matrices does not have this property [7]. Number $1-\sum_{n} F_{k, n}\left(t_{1}, t_{2}\right)$ is a probability that the realization of stochastic processes starting from the state $E_{k}$ at time $t_{1}$ reaches infinity before time $t_{2}$. For many infinity intensity matrices this probability is positive.

\section{The stochastic matrix of homogeneous stochastic processes}

For a homogeneous stochastic process the intensity matrix $\left[f_{k, n}(t)\right]_{k, n \in \mathrm{N}}$ is independent of time. It is a matrix $\left[a_{k, n}\right]_{k, n \in \mathrm{N}}$ of constant functions, whose integrals can be easily calculated. The $a_{k, n}$ are real numbers, positive for $k \neq n$ and negative for $k=n$, and $\sum_{n} a_{k, n}=0$ for all $k$.

The calculation of the stochastic matrix $\left[A_{k, n}(\Delta)\right]_{k, n \in \mathrm{N}}$ can be realised by the calculation of $A_{k, n}^{r}(\Delta)$ where $A_{k, n}(\Delta)=\sum_{r=0}^{\infty} A_{k, n}^{r}(\Delta)$ and:

$$
A_{k, n}^{r}(\Delta)=\sum_{m_{1} \neq k m_{2} \neq m_{1}} \ldots \sum_{m_{r-1} \neq m_{r-2}} H_{m_{r-1} \neq n}^{t+\Delta}\left(k, m_{1}, m_{2}, \ldots, m_{r-1}, n\right) .
$$

The $H_{t, r}^{t+\Delta}\left(k, m_{1}, m_{2}, \ldots, m_{r-1}, n\right)$ are given by formulas (21)-(23) after substitution $t_{1}=t$ anf $t_{2}=t+\Delta$.

For $r=0$ :

$$
H_{t, 0}^{t+\Delta}(k)=\exp \left(\int_{t}^{t+\Delta} a_{k, k} d s\right)=\exp \left(a_{k, k}(\Delta)\right)
$$

For $r=1$ :

$$
\begin{array}{r}
H_{t, 1}^{t+\Delta}(k, n)=\int_{t}^{t+\Delta} a_{k, n} \exp \left(\int_{t}^{x} a_{k, k} d s+\int_{x}^{t+\Delta} a_{n, n} d s\right) d x= \\
=\int_{t}^{t+\Delta} a_{k, n} \exp \left(a_{k, k}(x-t)+a_{n, n}(t+\Delta-x)\right) d x= \\
\left\{\begin{array}{l}
\frac{a_{k, n}}{a_{k, k}-a_{n, n}}\left(\exp \left(a_{k, k} \Delta\right)-\exp \left(a_{n, n} \Delta\right)\right) i f a_{k, k} \neq a_{n, n} \\
a_{k, n} \Delta \exp \left(a_{k, k} \Delta\right) \quad \text { if } a_{k, k}=a_{n, n}
\end{array}\right.
\end{array}
$$

For $r=2$ : 


$$
\begin{gathered}
H_{t, 2}^{t+\Delta}(k, n)= \\
\sum_{m} \int_{t}^{t+\Delta} \int_{x_{1}}^{t+\Delta} a_{k, m} a_{m, n} \exp \left(\int_{t}^{x_{1}} a_{k, k} d s+\int_{x_{1}}^{x_{2}} a_{m, m} d s+\int_{x_{2}}^{t+\Delta} a_{n, n} d s\right) d x_{2} d x_{1}= \\
\sum_{m} \int_{t}^{t+\Delta} \int_{x_{1}}^{t+\Delta} a_{k, m} a_{m, n} \exp \left(a_{k, k}\left(x_{1}-t\right)+a_{m, m}\left(x_{2}-x_{1}\right)+a_{n, n}\left(t+\Delta-x_{2}\right)\right) d x_{2} d x_{1}
\end{gathered}
$$

The double integral (named $I(m)$ ) must be calculated after consideration of all possible dependencies between $a_{k, k}, a_{m, m}$ and $a_{n, n}$.

If $a_{k, k}=a_{m, m}=a_{n, n}$ then:

$$
I(m)=\frac{a_{k, m} a_{m, n} \Delta^{2} \exp \left(a_{k, k} \Delta\right)}{2}
$$

If $a_{k, k}=a_{m, m}$ and $a_{m, m} \neq a_{n, n}$ then:

$$
I(m)=\frac{a_{k, m} a_{m, n} \Delta \exp \left(a_{k, k} \Delta\right)}{a_{k, k}-a_{n, n}}-\frac{a_{k, m} a_{m, n}\left[\exp \left(a_{k, k} \Delta\right)-\exp \left(a_{n, n} \Delta\right)\right]}{\left(a_{k, k}-a_{n, n}\right)^{2}}
$$

If $a_{k, k} \neq a_{m, m}$ and $a_{m, m}=a_{n, n}$ then:

$$
I(m)=\frac{a_{k, m} a_{m, n} \Delta \exp \left(a_{n, n} \Delta\right)}{a_{k, k}-a_{n, n}}-\frac{a_{k, m} a_{m, n}\left[\exp \left(a_{k, k} \Delta\right)-\exp \left(a_{n, n} \Delta\right)\right]}{\left(a_{k, k}-a_{n, n}\right)^{2}}
$$

If $a_{k, k}=a_{n, n}$ and $a_{k, k} \neq a_{m, m}$ then:

$$
I(m)=\frac{a_{k, m} a_{m, n} \Delta \exp \left(a_{k, k} \Delta\right)}{a_{k, k}-a_{m, m}}-\frac{a_{k, m} a_{m, n}\left[\exp \left(a_{k, k} \Delta\right)-\exp \left(a_{m, m} \Delta\right)\right]}{\left(a_{k, k}-a_{m, m}\right)^{2}}
$$

If $a_{k, k} \neq a_{m, m}$ and $a_{m, m} \neq a_{n, n}$ and $a_{k, k} \neq a_{n, n}$

$$
I(m)=\frac{a_{k, m} a_{m, n}}{\left(a_{k, k}-a_{m, m}\right)\left(a_{k, k}-a_{n, n}\right)\left(a_{m, m}-a_{n, n}\right)} \cdot \varphi\left(a_{k, k}, a_{m, m}, a_{n, n}\right)
$$

where

$$
\varphi\left(a_{k, k}, a_{m, m}, a_{n, n}\right)=\left(a_{k, k}-a_{n, n}\right) \exp \left(\left(a_{k, k}+a_{n, n}-a_{m, m}\right) \Delta\right)-
$$




$$
\left(a_{k, k}-a_{m, m}\right) \exp \left(a_{k, k} \Delta\right)-\left(a_{m, m}-a_{n, n}\right) \exp \left(a_{n, n} \Delta\right)
$$

Writting the formulas for any $r$ is impossible. The complicated form of these equations is the cause of the lack of them in the textbooks of probability.

\section{Conclusions}

This paper shows that an intensity matrix is more basic in the theory of continuous-time, Markov stochastic processes than a stochastic matrix. The intensity matrix generates a stochastic matrix and sometimes this matrix does not satisfy the condition: the sum of elements in each row equals one. Therefore, this condition is not necessary for stochastic matrices.

Although the form of equation (20) is not simple, it allows proving some theorems for Markov stochastic processes. This equation allows calculating a stochastic matrix by computer programs for given intensity matrices. Because series (20) is convergent, for any $\varepsilon>0$ there exists such $r_{s} \in \mathrm{N}$ that:

$$
\sum_{r=r_{s}}^{\infty} \sum_{m_{1} \neq k} \ldots \sum_{m_{r-1} \neq m_{r-2}} H_{m_{r-1} \neq n}^{t_{2}}\left(k, m_{1}, \ldots, m_{r-1}, n\right) \leq \varepsilon
$$

Therefore, a calculation of a stochastic matrix with willed accuracy is possible after finite steps.

Having state transition matrix we can calculate an ergodic coefficient, a limited matrix for a stochastic process probability distribution after long time (if ergodic coefficient is less than 1) and other characteristics of stochastic processes. Until now, this was not possible.

\section{References}

[1] Asmussen, S. R., Applied Probability and Queues, Springer-Verlag, New York, Berlin, Heidelberg, (2003).

[2] Chen, B.; Zhou, X.-H., A correlated random effects model for non-homogeneous Markov processes with nonignorable missingness, J. Multivar. Anal. 117 (2013), 1-13.

[3] Cheng-Chi Huang Non-homogeneous Markov chains and their applications, A Dissertation Submitted to the Graduate Faculty in Partial Fulfillment of The Requirements for the Degree of DOCTOR OF PHILOSOPHY. lova State University. (1977), http://lib.dr.iastate.edu/rtd

[4] Dessie, Z. G., Modeling of HIV/AIDS dynamic evolution using non-homogeneous semi-Markov process, SpringerPlus (2014) 3: 1-9. 3 (2014), 1-9.

[5] Di Biase, G.; Janssen, J.; Manca R., A Non-Homogeneous Continuous Time Semi-Markov Model for the Study of Accumulated Claim Process, Methodol. Comput. Appl. Probab. 12 (2010), 227-235.

[6] Janssen, J.; Manca, R., Numerical Solution of Non-Homogeneous Semi-Markov Processes in Transient Case, Method. Comput. Appl. Probab. 3 (2001), 171-293.

[7] Feller, W., An introduction to probability theory and its applications, vol. 1, John Willey and Sons, Inc. New York (1967).

[8] Latouche, G.; Ramaswami, V., Introduction to Matrix Analytic Methods in Stochastic Modelling, ASA-SIAM, (1999).

[9] Lucas, A.; Monteiro, A.; Smirnov, G. V. Nonparametric Estimation for Non-homogeneous Semi-Markov Processes: an Application to Credit Risk, Tinbergen Institute Discussion Paper. (2006), https://www.econstor.eu/bitstream/10419/86386/1/06-024.pdf

[10] Miranda, J. C. S.; Morettin P. A., Estimation of the intensity of non-homogeneous point processes via wavelets, Ann. Inst. Stat. Math. 63 (2011), 1221-1246.

[11] Reynaud-Bouret, P., Adaptive estimation of the intensity of inhomogeneous Poisson processes via concentration inequalities, Probab. Theory Relat. Fields 126 (2003), 103-153.

[12] Ruggeri, F. ; Ganesan, S., On Modeling Change Points in Non-Homogeneous Poisson Processes, Stat. Infer. Stoch. Processes 8 (2005), 311-329. 
[13] Sokó ł, M., A probability space of continuous-time discrete value stochastic process with Markov property, J. Adv. Math. 12 (2016), 5975-5989.

[14] Stenberg, F. Manca, R. Silvestrov, D., An Algorithmic Approach to Discrete Time Non-homogeneous Backward Semi-Markov Reward Processes with an Application to Disability Insurance, Methodol Comput Appl Probab. 9 (2007), 495-519.

[15] Syski, R., Passage Times for Markov Chains, IOS Press, (1992).

[16] Vidyasagar, M., Hidden Markov Processes : Theory and Applications to Biology, Princeton University Press., (2014).

[17] Yu, J. S.; Lin Y. K., Numerical path integration of a non-homogeneous Markov process, Inter. J. Non-Linear Mech. 39 (2004), 1493-1500.

\section{(c)}

This work is licensed under a Creative Commons Attribution 4.0 International License. 